\title{
Mitochondrial Dysfunction in Trauma-Related Coagulopathy: Is There Causality? Study Protocol for a Prospective Observational Study
}

\author{
Péter Jávor ${ }^{\mathrm{a}}$ Ferenc Rárosi ${ }^{\mathrm{b}}$ Tamara Horváth ${ }^{\mathrm{c}}$ László Török ${ }^{\mathrm{a}}$ \\ Petra Hartmann ${ }^{\mathrm{a}}$ \\ aDepartment of Traumatology, University of Szeged, Szeged, Hungary; ${ }^{\text {b}}$ Department of Medical Physics \\ and Informatics, University of Szeged, Szeged, Hungary; Institute of Surgical Research, University of Szeged, \\ Szeged, Hungary
}

\section{Keywords \\ Blood coagulation disorders - Blood platelet disorders . \\ Coagulopathy · Trauma induced · Hemorrhage · \\ Mitochondria}

\begin{abstract}
Hemorrhage control often poses a great challenge for clinicians due to trauma-induced coagulopathy (TIC). The pathogenesis of TIC is not completely revealed; however, growing evidence attributes a central role to altered platelet biology. The activation of thrombocytes and subsequent clot formation are highly energetic processes being tied to mitochondrial activity, and the inhibition of the electron transport chain (ETC) impedes on thrombogenesis, suggesting the potential role of mitochondria in TIC. Our present study protocol provides a guide to quantitatively characterize the derangements of mitochondrial functions in TIC. One hundred eleven severely injured (injury severity score $\geq 16$ ), bleeding trauma patients with an age of 18 or greater will be included in this prospective observational study. Patients receiving oral antiplatelet agents including cyclooxygenase-1 or adenosine diphosphate receptor inhibitors (aspirin, clopidogrel, prasugrel, and ticagrelor) will be excluded from the final
\end{abstract}

karger@karger.com www.karger.com/es

Karger!"
(C) 2021 The Author(s)

Published by S. Karger AG, Basel

This is an Open Access article licensed under the Creative Common Attribution-NonCommercial-4.0 International License (CC BY-NC) (http://www.karger.com/Services/OpenAccessLicense), applicable to the online version of the article only. Usage and distribution for commercial purposes requires written permission. analysis. Hemorrhage will be confirmed and assessed with computer tomography. Conventional laboratory markers of hemostasis such as prothrombin time and international normalized ratio will be measured and rotational thromboelastometry (ROTEM) will be performed directly upon patient arrival. Platelets will be isolated from venous blood samples and subjected to high-resolution fluororespirometry (Oxygraph-2k, Oroboros Instruments, Innsbruck, Austria) to evaluate the efficacy of mitochondrial respiration. Oxidative phosphorylation (OxPhos), coupling of the ETC, mitochondrial superoxide formation, mitochondrial membrane potential changes, and extramitochondrial $\mathrm{Ca}^{2+}{ }^{2+}$ movement will be recorded. The association between OxPhos capacity of platelet mitochondria and numerical parameters of ROTEM aggregometry will constitute our primary outcome. The relation between OxPhos capacity and results of viscoelastic assays and conventional markers of hemostasis will serve as secondary outcomes. The association of the OxPhos capacity of platelet mitochondria upon patient arrival to the need for massive blood transfusion and 24-h mortality will constitute our tertiary outcomes. Mitochondrial dysfunction and its importance in TIC are yet to be assessed for the deeper understanding of this common, life-threatening condition. Disclosure of mitochondria-mediated processes in 
thrombocytes may reveal new therapeutic targets in the management of hemorrhaging trauma patients, thereby leading to a reduction of potentially preventable mortality. The present protocol was registered to ClinicalTrials.gov on 12 August 2021, under the reference number NCT05004844.

(c) 2021 The Author(s).

Published by S. Karger AG, Basel

\section{Background}

The majority of potentially preventable mortality in trauma patients is related to bleeding [1-3]. Hemorrhage control often poses a great challenge for clinicians due to trauma-induced coagulopathy (TIC), a condition that is present in one-third of bleeding trauma patients [4]. Alterations in coagulation following severe injury were documented already in the 1960s [5]; however, a standard definition for TIC still does not exist [6]. TIC is characterized by dysfunctional clot formation and breakdown, impaired vascular homeostasis and is associated with increased risk of multiple organ failure and mortality $[7,8]$. Regarding the pathogenesis of TIC, the contribution of factor depletion and dysregulated fibrinolysis is clear; however, growing evidence attributes central role to altered platelet biology [9-11]. According to related studies, dysfunctional platelet aggregation can be identified with aggregometry assays in, approximately, $50 \%$ of trauma patients, entailing a higher risk for mortality $[12,13]$. Diminished platelet functions are suggested to be consequences of injury-induced early hyperactivation [10]; nevertheless, the mediators and pathways of the process are elusive, thus being subjects for further research [4, 14].

In the past decade, the presence of altered mitochondrial functions has been confirmed in the background of several diseases [15-17]. Furthermore, mitochondrial dysfunction of various cell types occurs also in traumarelated conditions such as hemorrhagic shock and traumatic brain injury [18-20]. As platelets are considered as central mediators in TIC, the understanding of mitochondria-mediated processes in thrombocytes may disclose new therapeutic targets in the management of severely injured patients [21]. It is already well known that the activation of thrombocytes and subsequent clot formation are highly energetic processes being tied to mitochondrial activity $[22,23]$. According to the literature, inhibition of the electron transport chain impedes on thrombogenesis [24-26], suggesting the potential role of mitochondria in TIC. Furthermore, the decreased physiological function of transfused platelets is believed to be a consequence of deteriorated mitochondrial respiration occurring already after 2 days of storage in blood-banked platelets [21, 27].

We hypothesize that mitochondrial dysfunction occurs in the platelets of trauma patients with TIC. We intend to quantitatively characterize the derangements of mitochondrial functions in TIC, and assess the relation between mitochondrial respiration and clinical markers of platelet function measured with aggregometry, viscoelastic tests, and conventional laboratory analysis.

\section{Methods}

Study Design

The present paper is a protocol for a single-center, prospective observational study that will be conducted at the University of Szeged, Szeged, Hungary. Our protocol complies with the Declaration of Helsinki and follows the Standard Protocol Items: Recommendations for Interventional Trials (SPIRIT) checklist (see online suppl. file. 1; see www.karger.com/doi/10.1159/000521670 for all online suppl. material).

\section{Patient Enrollment and Inclusion Criteria}

This prospective observational study will involve severely injured (injury severity score $\geq 16$ ) patients with bleeding confirmed with computer tomography, aged $\geq 18$ years, transported directly to the emergency department of the University of Szeged. Patients receiving oral antiplatelet agents including cyclooxygenase-1 or adenosine diphosphate (ADP) receptor (P2Y12) inhibitors (aspirin, clopidogrel, prasugrel, and ticagrelor) will be excluded from the final analysis. The study will be conducted for an estimated maximum of 36 months (between September 2021 and September 2024). Figure 1a (Protocol Flowchart) includes an overview on patient enrollment.

\section{Rotational Thromboelastometry}

In our present paper, rotational thromboelastometry (ROTEM) will be used to yield a comprehensive analysis of the hemostatic functions of study participants. ROTEM is a widely used point-ofcare tool providing rapid assessment of specific clotting pathways and platelet functions through viscoelastic assays and aggregometry $[6,28]$.

\section{Viscoelastic Assays}

Viscoelastic assays aid clinicians in choosing the appropriate blood products for patients with acute hemorrhage. Measurements require whole citrated blood to be transferred to a cylindrical cup, in which a pin performs an oscillating rotational movement. Until the blood remains in liquid state, the rotational movement is unrestricted. As soon as the blood starts the clotting process, the pin encounters increasing resistance due to rising clot firmness. Thus, the rotation of the pin is inversely proportional to clot firmness. An optical system detects the impedance of the rotation of the pin, and an integrated computer calculates the ROTEM curve and its numerical parameters. The instrument includes four measurement channels for four simultaneous assays from the following 5 test types: INTEM, EXTEM, APTEM, 


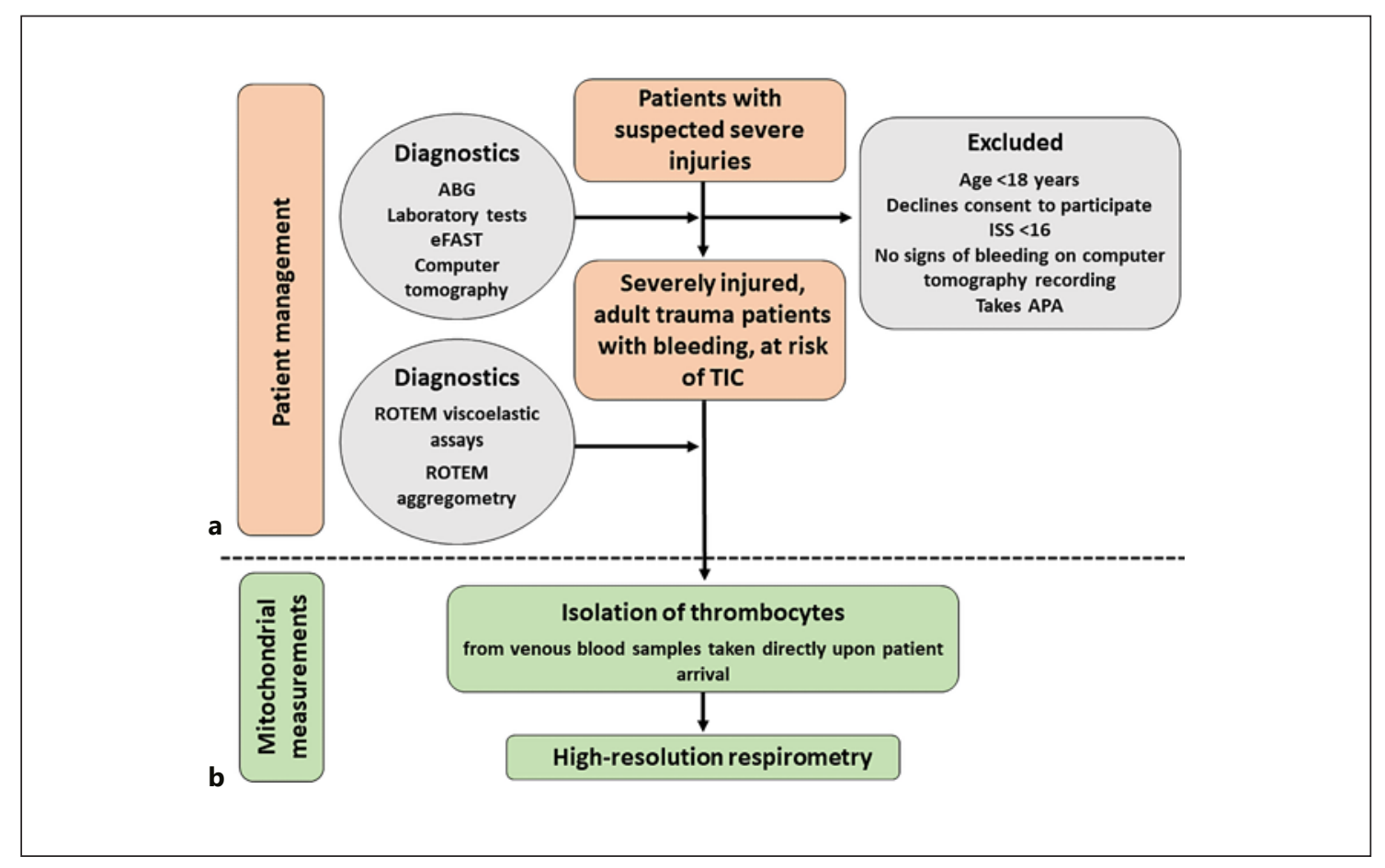

Fig. 1. Protocol flowchart. a Selection and management of study participants. Patients transported directly to the Emergency Department of the University of Szeged with suspected severe injuries undergo comprehensive diagnostics including ABG, laboratory tests (including $\mathrm{Hb}, \mathrm{Ht}, \mathrm{PLT}$, aPTT, prothrombin time (PT), and INR), extended focused assessment with sonography in trauma, and computer tomography. Severely injured (injury severity score $\geq 16$ ), adult trauma patients with confirmed bleeding will be enrolled into our study. Signed informed consent from patients or their surrogates will be required for inclusion. Taking antiplatelet agents entails exclusion from the analysis. The enrolled bleeding patients will undergo ROTEM viscoelastic tests and platelet ag-

FIBTEM, and HEPTEM. Coagulation can be activated intrinsically (INTEM) or extrinsically (EXTEM). The APTEM test uses fibrinolysis inhibitors aprotinin or tranexamic acid, thus comparing EXTEM to APTEM serves to detect hyperfibrinolysis. In the FIBTEM test, the contribution of platelets to clot formation is inhibited by cytochalasin-D; consequently, clotting depends solely on fibrin formation and polymerization. HEPTEM contains heparinase, thus serves to confirm the presence of heparin in the sample. Numerical parameters provided by ROTEM include clotting time (CT), clot formation time (CFT), speed of clot formation ( $\alpha$-angle), amplitude $10 \mathrm{~min}$ after CT (A10), maximum clot firmness (MCF), lysis index $30 \mathrm{~min}$ after CT (LI30), and maximum lysis (ML) [28-30].

\section{Aggregometry}

The ROTEM platelet module is an impedance aggregometer providing quantitative and qualitative information on platelet function in anticoagulated whole blood samples [31]. Blood is transferred into a cuvette containing a stirring pin and special elec- gregometry to provide a comprehensive description on coagulative functions. b Mitochondrial measurements. Thrombocytes will be isolated from venous blood samples taken upon patient arrival and subjected to high-resolution fluororespirometry to measure the efficacy of platelet mitochondrial respiration (OxPhos) and coupling of the mitochondrial ETC. Additionally, mitochondrial membrane potential changes and extramitochondrial $\mathrm{Ca}^{2+}$ movement will also be measured in the samples. ABG, arterial blood gas analysis; eFAST, extended focused assessment with sonography in trauma; ISS, injury severity score; APA, antiplatelet agent; Hb, hemoglobin; Ht, hematocrit; PLT, platelet count. trodes. After determining an impedance baseline, platelet aggregation is initiated with aggregating agents (ADP by ADPTEM, arachidonic acid by ARATEM, and thrombin activating peptide by TRAPTEM). The increase of electrical impedance is proportional to the number of platelets coating the electrodes by aggregation. Ultimately, a special software analysis displays the results on two channels, simultaneously [32]. The numerical values characterizing platelet aggregation are area under curve (AUC), maximum slope (MS), and amplitude at 6 min (A6).

Aggregometry with ROTEM can provide information on drugrelated and not-drug-related platelet dysfunction as well. In clinical practice, ADPTEM test is suitable for patients taking cyclooxygenase inhibitors such as acetylsalicylic acid. ARATEM is used for patients treated with ADP receptor antagonists such as clopidogrel, while TRAPTEM is the test of choice for patients taking GP IIb/IIIa receptor antagonists such as abciximab. Non-drug-induced platelet dysfunction may be detected on all tests; nevertheless, in case of TIC, TRAPTEM test is expected to display the derangements of platelet functions the most, prominently. 
Table 1. Documentation plan

\begin{tabular}{lll}
\hline & $\begin{array}{l}\text { Patient } \\
\text { arrival }\end{array}$ & $\begin{array}{c}\text { 24 h after } \\
\text { arrival }\end{array}$ \\
\hline Informed consent from surrogates & $\mathrm{X}$ & \\
Recording demographic data (age, sex) and comorbidities & $\mathrm{X}$ \\
eFAST & $\mathrm{X}$ & \\
Computer tomography & $\mathrm{X}$ \\
Listing and assessing all injuries & $\mathrm{X}$ & \\
Determining ISS & $\mathrm{X}$ \\
Assessment for eligibility & $\mathrm{X}$ \\
ABG (including BD and lactate) & $\mathrm{X}$ & \\
Laboratory testing (including Hb, Ht, PLT, aPTT, PT, INR) & $\mathrm{X}$ & $\mathrm{X}$ \\
ROTEM viscoelastic tests and aggregometry & $\mathrm{X}$ & \\
Isolation of thrombocytes from venous blood samples) & $\mathrm{X}$ & \\
High-resolution fluoroespirometry of platelet suspensions & $\mathrm{X}$ & \\
Recording MBT & & $\mathrm{X}$ \\
Recording 24-h mortality & & $\mathrm{X}$
\end{tabular}

Key measures of the protocol and their timing are shown. Informed consent will be obtained from patients or their surrogates upon admission. Imaging modalities will be used to assess bleeding and to aid the recognition of all injuries and determining ISS. Conventional laboratory tests, ROTEM, viscoelastic tests and aggregometry, and mitochondrial functional measurements will be performed. MBTs and 24-h mortality will be registered. eFAST, extended focused assessment with sonography in trauma; ISS, injury severity score; $A B G$, arterial blood gas analysis; $B D$, base deficit, $\mathrm{Hb}$, hemoglobin; $\mathrm{Ht}$, hematocrit; PLT, platelet count; thromboplastin time; PT, prothrombin time.

Recorded Variables

Demographic data and comorbidities of the participants will be documented ideally upon admission. In case of the identity of the patient is unknown, surrogates must be identified and contacted within $24 \mathrm{~h}$ to obtain missing information and informed consent for study participation. Upon patient arrival, conventional laboratory tests including hemoglobin, hematocrit, platelet count, activated partial thromboplastin time (aPTT), prothrombin time (PT), and international normalized ratio (INR) will be performed. Computer tomography and extended focused assessment with sonography in trauma will be used to detect internal bleeding. ROTEM viscoelastic assays and aggregometry will be carried out to assess coagulopathy. Clotting time, CFT, $\alpha$-angle, A10, MCF, LI30, and ML in INTEM, EXTEM, APTEM, and FIBTEM tests; AUC, MS, and A6 in TRAPTEM test will be documented. Massive blood transfusions (MBTs) and 24-h mortality will be recorded. The present study defines MBT, according to advanced trauma life support, as more than 10 units of transfused packed red blood cells within the first $24 \mathrm{~h}$ of admission or more than 4 units in $1 \mathrm{~h}$. The detailed documentation plan is demonstrated in Table 1.

\section{Mitochondrial Functional Measurements}

Platelets will be isolated from venous blood samples taken directly upon patient arrival. The efficacy of mitochondrial respiration (oxidative phosphorylation [OxPhos] and coupling of the mitochondrial electron transport chain) will be evaluated by highresolution fluoroespirometry (Oxygraph-2k, Oroboros Instruments, Innsbruck, Austria), after permeabilization of platelets. Mitochondrial superoxide formation, mitochondrial mem- brane potential changes, and extramitochondrial $\mathrm{Ca}^{2+}$ movement will also be assessed. Figure $1 \mathrm{~b}$ (protocol flowchart) demonstrates an overview on mitochondrial functional measurements.

\section{Data Management}

Only investigators named in the ethical approval are entitled to handle patient data. In attempt to trace data to an individual subject, a subject identification code list will be used. A personal identification number will be generated to identify the data of the participants and it will be present on all forms and documents. Electronic case report form (eCRF) will be applied. The principal investigator will ensure that the data in the eCRF are accurate, complete, and legible. Any missing or inconsistent recordings in the eCRFs will be requested from the responsible investigator using a data query form and will be corrected for each individual subject before clean file status is declared.

\section{Commitment to Data Sharing}

Our data management procedure complies with the FAIR principles (F: findable, A: accessible, I: interoperable, R: reusable). The source data and the individual patient records are stored in a secure hospital information system (MedSolution). The eCRFs in pdf format are sent to the Regional and Institutional Review Board of Human Investigations at the University of Szeged (Contact: office.rkeb@med.u-szeged.hu) with the annual periodic report of the study. These documents are not public, but the Regional and Institutional Review Board of Human Investigations at the University of Szeged reserves the right to decide in each individual case if the original eCRFs can be shared upon request. The study protocol is already entered prospectively into publicly accessible trial regis- 
try (ClinicalTrials.gov), and the authors declare that the results derived from the study will be published as a journal paper.

\section{Outcomes}

The association between OxPhos capacity of platelet mitochondria and numerical parameters of ROTEM aggregometry (AUC, MS, and A6 in TRAPTEM) will constitute our primary outcome. The relation between OxPhos and results of viscoelastic assays (CT, CFT, a-angle, A10, MCF, LI30, and ML in INTEM, EXTEM, APTEM, FIBTEM) and conventional markers of hemostasis (aPTT, PT, INR) will serve as secondary outcomes. The association of the OxPhos capacity of platelet mitochondria upon patient arrival to the need for MBT and 24-h mortality will constitute our tertiary outcomes.

\section{Statistical Methods}

Hypothesis

The alternative hypothesis for the primary outcome presumes an association (Pearson correlation at least 0.3 or larger) between OxPhos capacity of platelet mitochondria and thrombocyte aggregation (indicated by AUC, MS, and A6 in TRAPTEM test of ROTEM aggregometry).

\section{Sample Size Calculation}

Sample size calculation was performed with $G^{*}$ Power version 3.9.1.7 software. The estimation was based on the significance test for the correlation coefficient. We expect the magnitude of the correlation coefficient to be at least 0.3 . Thus, 111 subjects are needed to reject the null hypothesis that this correlation coefficient equals zero with the probability (power) of 0.95 . The significance level is $\alpha=0.05$.

\section{Statistical Analyses}

Statistical analyses will be performed using SPSS 25.0 (IBM Corporation, Chicago, IL, USA). $p$ values $p<0.05$ will be regarded as statistically significant. Continuous variables will be expressed as mean $\pm \mathrm{SD}, 95 \%$ confidence intervals. Significance test for the correlation coefficient will be applied for primary and secondary analyses.

To investigate the association between OxPhos capacity of platelet mitochondria and the need for MBT and 24-h mortality, respectively, ROC-analysis will be applied. No subgroup analyses are planned.

\section{Discussion}

TIC is a commonly occurring, severe condition contributing significantly to trauma-related mortality. Despite intensive research focus, the pathophysiology of TIC is still not completely understood, thus the appropriate, personalized therapy to each patient often cannot be delivered in emergency settings. Conventional laboratory markers of hemostasis such as aPTT, PT, and INR provide only limited information leading to the use of unnecessary or inappropriate blood products, thus resulting in suboptimal treatment and additional costs. Thromboelastometry (viscoelastic assays and aggregometry) overcomes several pitfalls of conventional laboratory tests; however, it also has its limitations. Experts claim that thromboelastometry is not performed on activated endothelium with physiological shear stress; therefore, it can hardly reflect in vivo clot formation, accurately [6]. Ultimately, in clinical reality, the transition from hypocoagulability to hypercoagulability is often impossible to detect, making it difficult to provide adequate therapy.

Disclosing and characterizing platelet mitochondrial dysfunction in TIC is necessary to deepen our understanding of the condition. As clot formation is tied to mitochondrial activity, further investigations may lead to new diagnostic tools and therapeutic targets in the management of TIC.

\section{Trial Status}

The study protocol has been registered to ClinicalTrials.gov under the identification number NCT05004844, on 12 August 2021. The study is currently in recruitment phase and can be identified unequivocally by the combination of ethical approval, protocols registration, and version numbers: 5500/2021-SZTE/NCT05004844/v1. Patient recruitment has started on 01 September 2021 and is expected to last till 01 September 2024.

\section{Statement of Ethics}

Our protocol complies with the Declaration of Helsinki and has been approved by the local medical ethics committee at the University of Szeged (Regional and Institutional Review Board of Human Investigations, Chairman: Prof. Dr. Tibor Wittmann) under reference number 5500/2021-SZTE. Our research studies patients that may be incapable of providing informed consent due to their condition; however, in these cases surrogates will be provided a consent form and information sheet describing the study in brief, and they will have the opportunity to consult one of the investigators. Personal information, photographs, or other material that might identify the participants will not be published. Personal data will not be given out without the permission of our patients or their surrogates.

\section{Conflict of Interest Statement}

The authors have no conflicts of interest to declare.

\section{Funding Sources}

The study was funded by the National Research Development and Innovation Office OTKA Research Grant FK138839; and by the Hungarian Academy of Sciences; János Bolyai Research Grant $\mathrm{BO} / 00605 / 21 / 5$. Funders did not influence the design and scientific content of our paper in any way. 


\section{Author Contributions}

Conceptualization: P.H., P.J.; Methodology: P.H., P.J.; Statistical methods, sample size calculation: F.R.; Writing - Original Draft Preparation: P.J.; Writing Review \& Editing: P.H., T.H.; Stylistic and grammatical revision: T.H.; Supervision: P.H., L.T.; Funding Acquisition: P.H.

\section{Data Availability Statement}

Not applicable. The study is in recruitment phase. Upon completion of the research, the final data set will be primarily available to the principal investigator (P.H.). The results will be shared with the scientific community through publication.

\section{References}

1 Eastridge BJ, Holcomb JB, Shackelford S. Outcomes of traumatic hemorrhagic shock and the epidemiology of preventable death from injury. Transfusion. 2019;59:1423-8.

2 Schoeneberg C, Schilling M, Hussmann B, Schmitz D, Lendemans S, Ruchholtz S. Preventable and potentially preventable deaths in severely injured patients: a retrospective analysis including patterns of errors. Eur J Trauma Emerg Surg. 2017;43:481-9.

3 Spahn DR, Rossaint R. Coagulopathy and blood component transfusion in trauma. $\mathrm{Br} J$ Anaesth. 2005; 95:130-9.

4 Kornblith LZ, Moore HB, Cohen MJ. Trauma-induced coagulopathy: the past, present, and future. J Thromb Haemost. 2019;17:85262.

5 Innes D, Sevitt S. Coagulation and fibrinolysis in injured patients. J Clin Pathol. 1964;17:113.

6 Moore EE, Moore HB, Kornblith LZ, Neal MD, Hoffman M, Mutch NJ, et al. Traumainduced coagulopathy. Nat Rev Dis Primers. 2021;7:30

7 Cohen MJ, Christie SA. New understandings of post injury coagulation and resuscitation. Int J Surg. 2016;33:242-5.

8 Brohi K, Singh J, Heron M, Coats T. Acute traumatic coagulopathy. J Trauma. 2003;54: 1127-30.

9 Kornblith LZ, Kutcher ME, Redick BJ, Calfee CS, Vilardi RF, Cohen MJ. Fibrinogen and platelet contributions to clot formation: implications for trauma resuscitation and thromboprophylaxis. J Trauma Acute Care Surg. 2014;76:255-3; discussion 62-3.

10 Wohlauer MV, Moore EE, Thomas S, Sauaia A, Evans E, Harr J, et al. Early platelet dysfunction: an unrecognized role in the acute coagulopathy of trauma. J Am Coll Surg. 2012;214:739-46.

11 Brown LM, Call MS, Margaret Knudson M, Cohen MJ, Trauma Outcomes G, Holcomb JB, et al. A normal platelet count may not be enough: the impact of admission platelet count on mortality and transfusion in severely injured trauma patients. J Trauma. 2011;71: S337-42.
12 Kutcher ME, Redick BJ, McCreery RC, Crane IM, Greenberg MD, Cachola LM, et al. Characterization of platelet dysfunction after trauma. J Trauma Acute Care Surg. 2012;73:13-9.

13 Jacoby RC, Owings JT, Holmes J, Battistella FD, Gosselin RC, Paglieroni TG. Platelet activation and function after trauma. J Trauma. 2001;51:639-47.

14 Savioli G, Ceresa IF, Caneva L, Gerosa S, Ricevuti G. Trauma-induced coagulopathy: overview of an emerging medical problem from pathophysiology to outcomes. Medicines. 2021;8(4).

15 Niyazov DM, Kahler SG, Frye RE. Primary mitochondrial isease and secondary mitochondrial dysfunction: importance of distinction for diagnosis and treatment. Mol Syndromol. 2016;7:122-37.

16 Panga V, Kallor AA, Nair A, Harshan S, Raghunathan S. Mitochondrial dysfunction in rheumatoid arthritis: a comprehensive analysis by integrating gene expression, proteinprotein interactions and gene ontology data. PLoS One. 2019;14:e0224632.

17 Norat P, Soldozy S, Sokolowski JD, Gorick CM, Kumar JS, Chae Y, et al. Mitochondrial dysfunction in neurological disorders: exploring mitochondrial transplantation. NPJ Regen Med. 2020;5:22.

18 Hiebert JB, Shen Q, Thimmesch AR, Pierce JD. Traumatic brain injury and mitochondrial dysfunction. Am J Med Sci. 2015;350:1328

19 Shi Y, Greven J, Guo W, Luo P, Xu D, Wang $\mathrm{W}$, et al. Trauma-hemorrhage stimulates immune defense, mitochondrial dysfunction, autophagy, and apoptosis in pig liver at $72 \mathrm{~h}$. Shock. 2021;55:630-9.

20 Warren M, Subramani K, Schwartz R, Raju R. Mitochondrial dysfunction in rat splenocytes following hemorrhagic shock. Biochim Biophys Acta Mol Basis Dis. 2017;1863:2526-33.

21 Saillant NN, Sims CA. Platelet dysfunction in injured patients. Mol Cell Ther. 2014;2:37.

22 Piguet PF, Vesin C, Da Kan C. Activation of platelet caspases by TNF and its consequences for kinetics. Cytokine. 2002;18:222-30.
23 Wolf BB, Goldstein JC, Stennicke HR, Beere H, Amarante-Mendes GP, Salvesen GS, et al. Calpain functions in a caspase-independent manner to promote apoptosis-like events during platelet activation. Blood. 1999;94: 1683-92.

24 Flierl U, Fraccarollo D, Widder JD, Micka J, Neuser J, Bauersachs J, et al. The nitric oxide donor pentaerythritol tetranitrate reduces platelet activation in congestive heart failure. PLoS One. 2015; 10:e0123621.

25 Wang Z, Cai F, Chen X, Luo M, Hu L, Lu Y. The role of mitochondria-derived reactive oxygen species in hyperthermia-induced platelet apoptosis. PLoS One. 2013;8:e75044.

26 Barile CJ, Herrmann PC, Tyvoll DA, Collman JP, Decreau RA, Bull BS. Inhibiting plateletstimulated blood coagulation by inhibition of mitochondrial respiration. Proc Natl Acad Sci U S A. 2012;109:2539-43.

27 Perales Villarroel JP, Figueredo R, Guan Y, Tomaiuolo M, Karamercan MA, Welsh J, et al. Increased platelet storage time is associated with mitochondrial dysfunction and impaired platelet function. J Surg Res. 2013;184: 422-9.

28 Božič Mijovski M. Advances in monitoring anticoagulant therapy. Adv Clin Chem. 2019; 90:197-213.

29 Wang IJ, Park SW, Bae BK, Lee SH, Choi HJ, Park SJ, et al. FIBTEM improves the sensitivity of hyperfibrinolysis detection in severe trauma patients: a Retrospective Study using thromboelastometry. Sci Rep. 2020;10:6980.

30 Hunt H, Stanworth S, Curry N, Woolley T, Cooper C, Ukoumunne O, et al. Thromboelastography (TEG) and rotational thromboelastometry (ROTEM) for trauma induced coagulopathy in adult trauma patients with bleeding. Cochrane Database Syst Rev. 2015; 2015:CD010438.

31 Koltai K, Kesmarky G, Feher G, Tibold A, Toth K. Platelet aggregometry testing: molecular mechanisms, techniques and clinical implications. Int J Mol Sci. 2017;18.

32 Nissen PH, Skipper MT, Hvas AM. Whole blood platelet aggregation determined by the ROTEM platelet equipment; reference intervals and stability. Platelets. 2020;31:215-20. 